\title{
Case Files of the Program in Medical Toxicology at Brown University: Amantadine Withdrawal and the Neuroleptic Malignant Syndrome
}

\author{
Eric J. Brantley, $M D^{a}$, Jamieson V. Cohn, $M D^{a}$, Kavita M. Babu, $M D^{b}$
}

aEmergency Medicine Residency, Brown University, Providence, RI

bProgram in Medical Toxicology, Department of Emergency Medicine, Warren J. Alpert School of Medicine, Brown University, Providence, RI

\section{CASE PRESENTATION}

A sixty-one-year old woman with a history of depression and idiopathic dystonia presented to the emergency department (ED) with fever, nausea, muscle rigidity, and redness in her extremities. The patient had seen various specialists for her dystonia during the preceding year and had tried multiple medication regimens, including benztropine and tizanidine. The patient described her home medications as tizanidine $2 \mathrm{mg}$ three times daily, diazepam $2.5 \mathrm{mg}$ daily, escitalopram $100 \mathrm{mg}$ daily, rosuvastatin $10 \mathrm{mg}$ daily and bupropion $300 \mathrm{mg}$ nightly.

At ED presentation her vital signs were: temperature $37.5^{\circ} \mathrm{C}$ (99.5F), blood pressure $157 / 76 \mathrm{mmHg}$, heart rate 105 beats per minute, respirations 24 per minute, and oxygen saturation of $100 \%$ on room air. Her physical examination was significant for diaphoresis, tachycardia, diffuse flushing, tremors in her upper and lower extremities, muscle rigidity, and hyperreflexia in the upper and lower extremities. The tremors were low amplitude and worse with intention, while the rigidity had cogwheeling features and was more notable in her lower extremities. Initial laboratory evaluation was significant for a creatine phosphokinase (CPK) of 1146 IU/L. Her electrocardiogram showed sinus tachycardia with normal QRS and QT intervals, and no evidence of acute ischemia.

Two months earlier, the patient's neurologist had started her on amantadine $100 \mathrm{mg}$ twice daily for dystonia. Three days prior to ED presentation, the patient abruptly stopped taking her aman- tadine on the advice of a pharmacist, secondary to bilateral lowerextremity edema. The following day, the patient presented to her primary care physician complaining of nausea, tremors, and diffuse flushing. The physician treated her for an allergic reaction with oral steroids and diphenhydramine. The patient's symptoms worsened over the next day with increasing tremors and rigidity, low-grade fever, nausea, and flushing, which prompted her presentation to the ED.

\section{What is the pharmacology of amantadine?}

Amantadine (Figure 1) is a synthetic, 10-carbon, polycyclic cage amine that was the first antiviral to be used in the prevention and treatment of influenza. The proposed mechanism of amantadine's efficacy against influenza is blockade of the viral M2 channel and subsequent inhibition of viral uncoating. However, its modern use against influenza has been limited by resistance, as well as the incidence of adverse central nervous system (CNS) effects [1]. In 1968, a patient noted significant improvement in her symptoms of Parkinson's disease (PD) after treatment with amantadine for influenza. This observation was confirmed in a small pilot study, then in a much larger trial that made amantadine part of the standard treatment regimen for PD. The mechanism of amantadine's amelioration of parkinsonian-induced tremors is unclear; however, proposed mechanisms include action at nigrostriatal neurons resulting in increased release or decreased reuptake of dopamine [2-4]. Additionally, amantadine decreases cholinergic tone, which may increase the affinity of D2 receptors for dopamine [5]. Amantadine

Keywords: amantadine withdrawal, rigidity, neuroleptic malignant syndrome

Note: There was no outside funding of any kind used for this study.

Acknowledgment: The authors gratefully acknowledge the contribution of Dr. Jason B. Hack to the preparation of this manuscript. Corresponding author: Kavita M. Babu, MD, Department of Emergency Medicine, 593 Eddy Street, Providence, RI 02903. Email: kavitambabu@gmail.com 


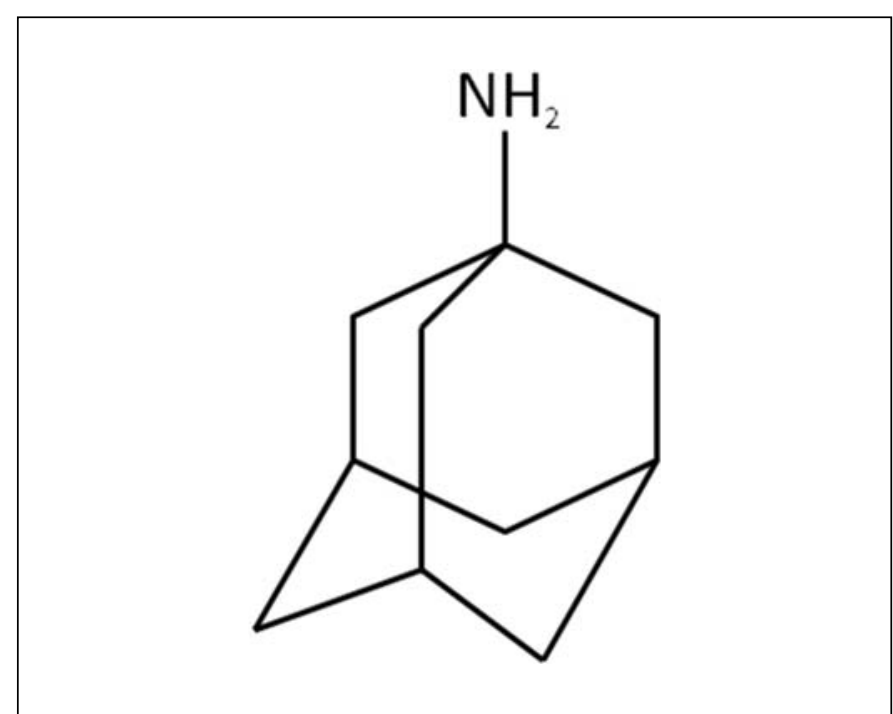

Figure 1: Structure of amantadine.

also functions as an NMDA (N-methyl-D-aspartic acid) receptor antagonist, inhibiting the excitatory glutaminergic pathways that play a role in generating the dyskinesias induced by levodopa in PD patients [6].

Amantadine hydrochloride is rapidly absorbed after oral ingestion, with approximately $90 \%$ bioavailability $[7,8]$. The average time to peak plasma concentration is $3.3 \pm 1.5$ hours [9]. Amantadine is $59-67 \%$ protein bound. Estimates of the volume of distribution of amantadine vary significantly, ranging from 3 to $10 \mathrm{~L} / \mathrm{kg}$ in various reports [8-10].

The majority of amantadine is excreted unchanged in the urine, although several amantadine metabolites have been identified. Approximately $5-15 \%$ of the ingested dose is excreted as the $\mathrm{N}$-acetylated form, acetylamantadine; the role of this metabolite in both therapeutics and toxicity is unknown [9]. The elimination half-life of amantadine ranges from 12 to 18 hours in healthy adults [8]. Multiple factors, including age, sex, renal insufficiency, and urine $\mathrm{pH}$, can affect time to elimination. Corrected for body mass index, men eliminate amantadine 1.5 times more quickly than women, and amantadine tends to be more rapidly excreted at acidic urine $\mathrm{pH}$ [9]. In elderly patients, or patients with a creatinine clearance below $40 \mathrm{ml} / \mathrm{min}$ per $1.73 \mathrm{~m}^{2}$, the elimination half-life may double or triple. In a cohort of patients on chronic hemodialysis, the elimination half-life increased to 8 days [9]. Given its high volume of distribution, amantadine elimination is negligibly affected by hemodialysis.

\section{What is amantadine's role in the treatment of Parkinson's disease and other movement disorders?}

Parkinson's disease results from decreased CNS dopaminergic tone and is characterized by bradykinesia, rigidity, and tremor. Loss of dopaminergic neurons in the substantia nigra leads to decreased excitation of the motor cortex and the characteristic features of PD.
The treatment of PD focuses on medications that increase dopaminergic tone (levodopa/carbidopa, bromocriptine), amantadine, anticholinergic agents, and monoamine oxidase-B inhibitors [11]. While levodopa remains one of the mainstays of PD therapy, patients on long-term levodopa therapy often develop worsening dyskinesias that frequently respond to treatment with amantadine.

While amantadine has been demonstrated to be effective in the treatment of both PD- and levodopa-induced dyskinesias, this benefit is maximal during the first month of treatment. By 3 to 8 months, the improvement in dyskinesia decreases or disappears in most patients [12]. As a result, many patients on amantadine therapy for PD will need to have this medication stopped for lack of efficacy within months of initiation.

Our patient did not carry a formal diagnosis of PD. However, amantadine has been used to treat a wide variety of other motor and tone abnormalities, including Huntington's chorea and idiopathic dystonias.

\section{Has a syndrome of amantadine withdrawal been described?}

A collection of symptoms resulting from abrupt amantadine discontinuation has been described [13-15]. These symptoms include varying degrees of tremor; muscle rigidity; autonomic instability (manifested by tachycardia, hypertension, and diaphoresis); delirium; elevation of serum CPK; and in some cases, fever. Similar symptoms have been noted after the rapid cessation of levodopa or bromocriptine [16-18]. The severity of symptoms and the presence of fever are important distinctions in classifying patients along a spectrum of amantadine withdrawal from worsening tremor to neuroleptic malignant syndrome (NMS).

In several cases of amantadine withdrawal-induced NMS, the patients had been treated with amantadine for prolonged periods of time; however, NMS has occurred after withdrawal of amantadine treatment after as few as 5 days $[15,19]$. While there have been fatalities associated with withdrawal of dopaminergic agonists, in our review of the medical literature we were unable to find any fatal case reports of amantadine withdrawal [20]. Despite the structural similarity of memantine (Namenda, Forest Pharmaceuticals, New York, NY) to amantadine, withdrawal symptoms have not been reported after memantine cessation.

\section{Does this patient have NMS?}

Neuroleptic malignant syndrome refers to an idiosyncratic, potentially life-threatening constellation of symptoms that has been described as progressing from mental status changes to motor symptoms, then hyperpyrexia and autonomic instability [21]. Most commonly described after initiation of neuroleptics or rapid dose escalation, the incidence of NMS has been approximated to be between 0.02 and $2.44 \%$ of patients receiving neuroleptic drugs [22]. In the majority of cases, NMS occurs within 2 weeks of neuroleptic initiation, and virtually all cases occur within 1 month after the last change in medications. In $16 \%$ of cases, symptoms may occur acutely, within 24 hours of neuroleptic administration [23]. While NMS was initially described after 
the use of older, "typical" antipsychotics like haloperidol, it has been associated with the atypical antipsychotics including clozapine, olanzapine, quetiapine, risperidone and ziprasidone $[24,25]$. Additionally, NMS has been described after use of antiemetics such as metoclopramide and promethazine, which also have antidopaminergic properties.

In addition to the use of antidopaminergic drugs, it is crucial to realize that withdrawal of prodopaminergic medications may also lead to NMS. A history of a patient's current medications may miss the agents that were withdrawn within the recent past, and lead to a missed diagnosis.

Recent data suggest a mortality rate of $10 \%$ in diagnosed NMS [26]. This figure has improved dramatically from original mortality rates of $76 \%$ in the 1960 s, which may be attributed to improved recognition, better supportive care, or increasing use of atypical antipsychotics [27]. Various published diagnostic criteria for NMS exist, and patients may fulfill criteria for NMS under one set, while unable to satisfy others [28]. The commonly cited DSM IV-TR criteria for diagnosis of NMS describe presence of 3 major criteria, including severe muscle rigidity and fever in the setting of neuroleptic use, with at least 2 minor criteria in the absence of medical or psychiatric conditions that could explain similar symptoms (Table 1). In contrast, the Levenson criteria allow the diagnosis of NMS with the presence of either rigidity or fever with appropriate history and the presence of additional minor criteria (Table 2).

Fever is frequently cited as a necessary element in the diagnosis of NMS. Dopamine is an important part of temperature regulation; stimulation of central dopamine receptors results in hypothermia, while central dopaminergic blockade or depletion leads to hyperthermia [29]. For this reason, most diagnostic cri-

Table 1: DSM IV-TR Diagnostic Criteria for Neuroleptic Malignant Syndrome* [53].

\begin{tabular}{|c|c|}
\hline \multirow[t]{3}{*}{ Major criteria } & Elevated temperature \\
\hline & Muscle rigidity \\
\hline & Setting of neuroleptic use \\
\hline \multirow[t]{10}{*}{ Minor criteria } & Diaphoresis \\
\hline & Dysphagia \\
\hline & Tremor \\
\hline & Incontinence \\
\hline & Altered level of consciousness \\
\hline & Mutism \\
\hline & Tachycardia \\
\hline & Hypertension or labile blood pressure \\
\hline & Elevated white blood cell count \\
\hline & Evidence of muscle injury (e.g., elevated CPK) \\
\hline Exclusions & $\begin{array}{l}\text { No other evident medical or psychiatric etiology } \\
\text { for symptoms }\end{array}$ \\
\hline
\end{tabular}

*Diagnosis requires all 3 major criteria and at least 2 minor criteria.
Table 2: Levenson diagnostic criteria for neuroleptic malignant syndrome. [34].

\begin{tabular}{ll}
\hline Major criteria & Elevated temperature \\
\hline Muscle rigidity \\
\hline Elevated CPK \\
\hline Altered mental status \\
\hline Blood pressure abnormalities \\
\hline Tachycardia \\
\hline Tachypnea \\
\hline Diaphoresis \\
\hline Elevated white blood cell count \\
\hline
\end{tabular}

*Diagnosis requires supportive clinical history and all 3 major criteria, or 2 major and 4 minor criteria.

teria for NMS describe a requirement for hyperpyrexia (temperature $>38^{\circ} \mathrm{C}$ ). However, several authors have described cases identical to NMS, except without fever [30-33]. Our patient never had a documented temperature above $38^{\circ} \mathrm{C}$; however, the history of subjective fever, as well as dysautonomia, altered mental status, and rigidity in the setting of amantadine withdrawal are highly suggestive of NMS, and satisfy the Levenson criteria for diagnosis in the setting of an elevated CPK [34]. It thus appears that a spectrum of disease associated with decreased dopaminergic tone exists and that it is not an "all or nothing" phenomenon. Neuroleptic malignant syndrome may simply contain the subset of patients that are most severely symptomatic. Arguably, the answer is also of little clinical importance as long as amantadine withdrawal is recognized and treatment initiated.

\section{What is the pharmacologic basis for the symptoms seen with NMS?}

Central dopaminergic blockade (at D2 receptors) or dopamine depletion have been postulated as the primary mechanisms for development of neuroleptic malignant syndrome [29]. While the exact mechanism of neuroleptic action is unknown, these agents are thought to antagonize the dopamine receptors (D1-4), as well as 5HT2 receptors [35]. Withdrawal of prodopaminergic agents causes similar symptoms. Decreased dopaminergic tone produces motor symptoms similar to parkinsonism, including tremor and rigidity. Dopamine also plays a role in temperature regulation, as described above.

\section{How should amantadine withdrawal or NMS be treated?}

For any patient presenting with symptoms of amantadine withdrawal or NMS, initial treatment must begin with attention to airway, breathing, circulation, level of consciousness, and correction of abnormal vital signs. Even when these causes are suspected, the basic approach to altered mental status should include a bedside glucose determination, volume resuscitation, and oxygen therapy 
as indicated, along with a vigorous search for other etiologies. Hyperthermia resulting from agitation and muscle rigidity requires urgent sedation with benzodiazepines, rapid cooling, and consideration of focused therapy with dopaminergic agents or dantrolene sodium (discussed below). Intubation and paralysis may be required in the patient with intractable hyperthermia. Patients who succumb from NMS typically die from complications of extreme hyperthermia including rhabdomyolysis, acute tubular necrosis, and complications of renal failure, dysrhythmias, and cardiovascular collapse secondary to intractable metabolic acidosis [36-38]. Renal failure has been associated with a mortality rate of 50\% [38]. Patients with suspected NMS should have screening laboratory analyses including complete blood count, serum chemistries, CPK, lactic acid, arterial or venous blood gas to establish $\mathrm{pH}$, a coagulation profile, and an electrocardiogram. A search for other etiologies of symptoms, including computed tomography of the brain and lumbar puncture, should be considered in patients with significant alteration in mental status.

Benzodiazepines can be used as adjunctive therapy to improve motor symptoms, and should be considered an important and safe part of supportive care. The causative neuroleptic must be withdrawn. In cases of suspected NMS secondary to medication withdrawal, the discontinued agent should be reinstituted. Additionally, other antidopaminergic medications must be carefully avoided, including antiemetics like promethazine and metoclopramide.

\section{What drugs and other therapies are used to treat NMS?}

While several medications are generally accepted as beneficial in decreasing mortality and reversing symptoms in patients with NMS, little data exist to definitively prove their benefit or relative efficacy. Nonetheless, dantrolene sodium, bromocriptine, and amantadine are routinely used in the treatment of NMS. Additionally, electroconvulsive therapy (ECT) has been successfully used to treat NMS. A retrospective case-control analysis by Sakkas et al. demonstrated a mortality rate of $21 \%$ in patients who did not receive either ECT or any of the above drug treatments. Dantrolene sodium alone reduced the death rate to $8.6 \%$, bromocriptine to $7.8 \%$, and amantadine to $6 \%$. In this series, combined therapy with pharmacologic agents had a higher mortality rate than single agents, which may have reflected increased severity of NMS in patients given multiple drugs. As no severity scores were provided, it is impossible to draw definitive conclusions [39]. Regardless, the study is highly suggestive of the benefit of any of these agents in NMS, even though it is unable to provide insight into which agents work better or the optimal combination. In cases of NMS from dopaminergic agent withdrawal, multiple case reports describe improvement of symptoms with reinstitution of the discontinued medication $[23,40]$.

Dantrolene is a direct skeletal muscle relaxant commonly used for malignant hyperthermia. In NMS, dantrolene works by reducing rigidity and concomitant hyperthermia, and may not be the most effective treatment modality in patients without significant hyperpyrexia [41]. Multiple dosing schedules have been described for dantrolene sodium in NMS. Administration has been recommended at $1-2.5 \mathrm{mg} / \mathrm{kg}$ intravenously; if symptoms improve, the patient can receive $1 \mathrm{mg} / \mathrm{kg}$ every 6-12 hours for an additional 48 hours, followed by $1 \mathrm{mg} / \mathrm{kg}$ per day for 8 days $[41,42]$. Milder symptoms may be treated with an oral regimen of 50-200 mg daily [36]. Dosing should not exceed $10 \mathrm{mg} / \mathrm{kg}$ per day in order to avoid hepatotoxicity [36]. While dantrolene has been demonstrated to be effective in the treatment of NMS, it is not specifically targeted at the proposed etiology for development of NMS.

Bromocriptine and amantadine both have dopaminergic effects; either may be used in the treatment of NMS. Bromocriptine is given at doses of $2.5-10 \mathrm{mg}$ orally every 6 hours and may be titrated up to a total dose of $40 \mathrm{mg} /$ day [36]. Amantadine is usually given at $100-200 \mathrm{mg}$ /day orally, but may be titrated up to 200 mg every 12 hours [37]. In general, once these medications are started, they should be continued for 10-14 days and then slowly tapered (Table 3). If these medications are tapered too quickly, NMS symptoms may recur [43].

\begin{tabular}{ll}
\hline Table 3: Treatment Regimens for NMS \\
\hline Medication & Dosing and duration of therapy* \\
\hline Amantadine & $\begin{array}{l}100-200 \mathrm{mg} \text { PO bid until symptoms resolve, } \\
\text { then taper over } 10-14 \text { days }\end{array}$ \\
\hline Bromocriptine & $\begin{array}{l}2.5-10 \mathrm{mg} \text { PO tid-qid until symptoms resolve, } \\
\text { then taper over } 10-14 \text { days }\end{array}$ \\
\hline Dantrolene sodium & $\begin{array}{l}1-2.5 \mathrm{mg} / \mathrm{kg} \text { hours IV, then } 1 \mathrm{mg} / \mathrm{kg} \mathrm{q} 6 \mathrm{~h} \mathrm{x} \\
48 \text { hours, then taper over } 3-10 \text { days }\end{array}$
\end{tabular}

Electroconvulsive therapy

*If treatment is tapered too rapidly, symptoms may recur.

ECT has also been reported to be effective treatment for NMS, particularly for cases in which medical management has failed. Additionally, malignant catatonia, characterized by rigidity and hyperthermia in patients with a history of psychosis, can be impossible to distinguish clinically from NMS. Some unique features of malignant catatonia include a prodrome of increasing agitation and psychosis. These patients are often taking neuroleptics, and authors have described the two syndromes as indistinguishable in up to $20 \%$ of patients [37]. The treatment for malignant catatonia is ECT; this treatment may have significant benefit in undifferentiated patients. One potential risk of ECT reported in NMS is development of cardiovascular complications, including ventricular dysrhythmias [44].

\section{What other toxicologic conditions should be included in the differential diagnosis of NMS?}

The constellation of rigidity, hyperthermia, dysautonomia, and altered mental status can be seen in serotonin syndrome, malignant hyperthermia, baclofen withdrawal, and lithium toxicity. 
A complete medication history remains the critical step in differentiating these conditions, as focused therapy for each of these disorders is unique. All of them share the potential for severe acidosis, rhabdomyolysis, and multisystem organ failure as a consequence of involuntary muscle activity and hyperpyrexia.

Serotonin syndrome has been described as a clinical triad of autonomic hyperactivity, neuromuscular abnormalities, and alteration in mental status changes that can be clinically similar to NMS; however, the diagnosis of serotonin syndrome hinges on the administration of a serotonergic medication within the preceding 5 weeks [45]. While the patient reported here was maintained on both bupropion and escitalopram, the medications had been maintained at stable doses over months, and the patient denied exposure to new over-the-counter medications or dietary supplements. The treatment of serotonin syndrome includes supportive care with intravenous fluids and benzodiazepines, antidotal therapy with cyproheptadine and, in severe cases, intubation and neuromuscular paralysis.

One description of amantadine-induced serotonin syndrome has been published in a patient with renal failure; however, the pharmacology of this case report is suspect as amantadine has not been described as a proserotonergic medication. Instead, this report describes muscle rigidity, tremor, fever, and autonomic instability consistent with the patient's history of recent changes in his antiparkinsonian regimen [46].

Malignant hyperthermia is caused by an inherited mutation of the ryanodine receptor in the sarcoplasmic reticulum of skeletal muscle [47]. Occurring just minutes after the administration of inhalational anesthetics or depolarizing neuromuscular blockers, malignant hyperthermia is characterized by extreme rigidity, hyperpyrexia, tachycardia, hypotension, and sometimes cyanosis [48]. The treatment of malignant hyperthermia includes supportive care and focused treatment with dantrolene, an inhibitor of sarcoplasmic calcium release.

Baclofen withdrawal is characterized by fever, mental status changes, spasticity, autonomic instability and seizures in the setting of rapid discontinuation of baclofen. This can occur with either intentional cessation of treatment, or in many cases, mechanical malfunction of implantable intrathecal pumps. Baclofen is an agonist of $\mathrm{GABA}_{\mathrm{A}}$ receptors that leads to downregulation of spinal inhibitory pathways with chronic use. When baclofen is stopped suddenly, patients develop symptoms consistent with unopposed excitatory CNS and peripheral nervous system tone, including seizures, motor abnormalities, and hyperpyrexia described above [49]. Temperatures as high as 43C have been reported in this setting. The treatment of baclofen withdrawal is reintroduction of baclofen, with supportive care, cooling, intubation, and paralysis as required for uncontrolled motor activity, hyperpyrexia, and lactic acidosis.

Other toxicologic conditions that may incompletely reproduce our patient's symptoms include Latrodectus and Centruroides envenomations, lithium toxicity, central anticholinergic toxicity, toxin-induced parkinsonism, strychnine, and sympathomimetics. Important nontoxicologic considerations include malig- nant catatonia, encephalitis, intracerebral hemorrhage, and heat stroke.

\section{What are common adverse effects of amantadine use?}

Amantadine is generally well-tolerated; however, several adverse effects have been described, including depression, anxiety, confusion, somnolence, decreased visual acuity, and livedo reticularis [9]. Bilateral lower-extremity edema is a well-described adverse effect in antiparkinsonian pharmacology, which has been reported with amantadine use, as well as dopamine agonists like pergolide and bromocriptine [50,51]. Risk factors for the development of edema include female gender, advanced age, diabetes, and cardiac disease. The degree of dependent edema can vary, and in some patients can lead to significant difficulty with ambulation. The edema typically resolves with discontinuation of the causative drug [52]. In our patient, the attribution of her edema to amantadine therapy was likely accurate; however, the rapid discontinuation of her medication led to her presenting symptoms.

\section{How should amantadine be discontinued?}

Although it is clear that motor symptoms, delirium, and NMS may occur with discontinuation of amantadine, it is unclear how quickly providers can stop the medication safely. No studies have demonstrated a safe time course for discontinuing amantadine. In one case series, patients on long-term amantadine therapy experienced delirium and hallucinations that began anywhere from 7 to 14 days after decreasing amantadine doses; these 3 patients were all in the midst of prolonged amantadine tapers [15]. Given the uncertainty in providing a safe withdrawal interval, tapering should also be done under close supervision of a neurologist. The patient must be givern strict instructions to seek medical attention if signs of worsening motor or cognitive dysfunction or NMS occur.

\section{CASE CONCLUSION:}

The clinical diagnosis in the ED was neuroleptic malignant syndrome secondary to amantadine withdrawal, although serotonin syndrome and infection remained on the differential diagnosis. The patient was given $2 \mathrm{~L}$ of normal saline, $1 \mathrm{mg}$ of lorazepam, $4 \mathrm{mg}$ of ondansetron, and $100 \mathrm{mg}$ of amantadine in the ED, and was transferred to the intensive care unit (ICU) for further monitoring and supportive care. In the ICU, the patient received aggressive intravenous fluid resuscitation and her serum CPK was monitored daily. Her peak CPK was 3322 IU/L. The elevated CPK was attributed to her muscle rigidity, given its corresponding rise with amantadine withdrawal and fall with drug reintroduction and symptomatic improvement. Another consideration included myositis secondary to her lipid-lowering agent; however, the acuity of presentation made this etiology less likely. The patient was restarted on amantadine $100 \mathrm{mg}$ twice a day, but her antidepressants were held. She was given lorazepam and ondansetron as needed for agitation and nausea, respectively. Throughout her 
ICU stay, the patient demonstrated hypertension with systolic blood pressures reaching $200 \mathrm{mmHg}$, which was difficult to control. She was discharged from the ICU on hospital day 5 after her symptoms had improved; her CPK at discharge had decreased to $307 \mathrm{IU} / \mathrm{L}$. She continued with amantadine $100 \mathrm{mg}$ twice a day as an outpatient with plans to taper her amantadine under the supervision of a neurologist.

The authors have no potential financial conflicts of interest to report.

\section{REFERENCES}

1. De Clercq E. Antiviral agents active against influenza A viruses. Nat Rev Drug Discov 2006;5(12):1015-1025.

2. Geldenhuys WJ, Malan SF, Bloomquist JR, et al. Pharmacology and structure-activity relationships of bioactive polycyclic cage compounds: a focus on pentacycloundecane derivatives. Med Res Rev 2005;25(1):21-48.

3. Mizoguchi K, Yokoo H, Yoshida M, et al. Amantadine increases the extracellular dopamine levels in the striatum by reuptake inhibition and by N-methyl-D-aspartate antagonism. Brain Res 1994;662(1-2):255-258.

4. Danysz W, Parsons CG, Kornhuber J, et al. Aminoadamantanes as NMDA receptor antagonists and antiparkinsonian agents-preclinical studies. Neurosci Biobehav Rev 1997;21(4): $455-468$.

5. Paci C, Thomas A, Onofrj M: Amantadine for dyskinesia in patients affected by severe Parkinson's disease. Neurol Sci 2001;22(1):75-76.

6. Snow BJ, Macdonald L, McAuley D, et al. The effect of amantadine on levodopa-induced dyskinesias in Parkinson's disease: a double-blind, placebo-controlled study. Clin Neuropharmacol 2000;23(2):82-85.

7. Aoki FY, Sitar DS. Clinical pharmacokinetics of amantadine hydrochloride. Clin Pharmacokinet 1988;14(1):35-51.

8. Cedarbaum JM. Clinical pharmacokinetics of antiparkinsonian drugs. Clin Pharmacokinet 1987;13(3):141-178.

9. Amantadine [package insert]. Generic. 1988.

10. Wu MJ, Ing TS, Soung LS, et al. Amantadine hydrochloride pharmacokinetics in patients with impaired renal function. Clin Nephrol 1982;17(1):19-23.

11. Rao SS, Hofmann LA, Shakil A: Parkinson's disease: diagnosis and treatment. Am Fam Physician 2006;74(12): 2046-2054.

12. Thomas A, Iacono D, Luciano AL, et al. Duration of amantadine benefit on dyskinesia of severe Parkinson's disease. J Neurol Neurosurg Psychiatry 2004;75(1):141-143.

13. Simpson DM, Davis GC: Case report of neuroleptic malignant syndrome associated with withdrawal from amantadine. Am J Psychiatry 1984;141(6):7967.

14. Toru M, Matsuda O, Makiguchi K, et al. Neuroleptic malignant syndrome-state following a withdrawal of antiparkinsonian drugs. J Nerv Ment Dis 1981;169(5):324-327.
15. Factor SA, Molho ES, Brown DL: Acute delirium after withdrawal of amantadine in Parkinson's disease. Neurology 1998;50(5):1456-1458.

16. Figa-Talamanca L, Gualandi C, Di Meo L, et al. Hyperthermia after discontinuance of levodopa and bromocriptine therapy: impaired dopamine receptors a possible cause. Neurology 1985;35(2):258-261.

17. Gibb WR, Griffith DN. Levodopa withdrawal syndrome identical to neuroleptic malignant syndrome. Postgrad Med J 1986;62(723):59-60.

18. Friedman JH, Feinberg SS, Feldman RG. A neuroleptic malignantlike syndrome due to levodopa therapy withdrawal. JAMA 1985;254(19):2792-2795.

19. Ito T, Shibata K, Watanabe A, et al. Neuroleptic malignant syndrome following withdrawal of amantadine in a patient with influenza A encephalopathy. Eur J Pediatr 2001;160(6):401.

20. Sechi GP, Tanda F, Mutani R. Fatal hyperpyrexia after withdrawal of levodopa. Neurology 1984;34(2):249-251.

21. Velamoor VR, Norman RM, Caroff SN, et al. Progression of symptoms in neuroleptic malignant syndrome. J Nerv Ment Dis 1994;182(3):168-173.

22. Khan M, Farver D. Recognition, assessment and management of neuroleptic malignant syndrome. S D J Med 2000;53(9):395-400.

23. Caroff S, Mann S. Neuroleptic Malignant Syndrome. Psychopharmacol Bull 1988; 24:25-29.

24. Ananth J, Parameswaran S, Gunatilake S, et al. Neuroleptic malignant syndrome and atypical antipsychotic drugs. J Clin Psychiatry 2004;65(4):464-470.

25. Murty RG, Mistry SG, Chacko RC. Neuroleptic Malignant Syndrome with ziprasidone. J Clin Psychopharmacol 2002;22(6):624-626.

26. Addonizio G, Susman VL, Roth SD. Neuroleptic malignant syndrome: review and analysis of 115 cases. Biol Psychiatry 1987;22(8):1004-1020.

27. Shalev A, Hermesh H, Munitz H. Mortality from neuroleptic malignant syndrome. J Clin Psychiatry 1989;50(1): $18-25$.

28. Caroff SN, Mann SC, Campbell EC. Atypical Antipsychotics and Neuroleptic Malignant Syndrome. Psychiatric Annals 2000;30(5):314-324.

29. Mann SC, Caroff SN, Fricchione G, et al. Central Dopamine Hypoactivity and the Pathogenesis of Neuroleptic Malignant Syndrome. Psychiatric Annals 2000;30(5):363-374.

30. Hynes AF, Vickar EL. Case study: neuroleptic malignant syndrome without pyrexia. J Am Acad Child Adolesc Psychiatry 1996;35(7):959-962.

31. Peiris DT, Kuruppuarachchi K, Weerasena LP, et al. Neuroleptic malignant syndrome without fever: a report of three cases. J Neurol Neurosurg Psychiatry 2000;69(2):277-278.

32. Rodriguez OP, Dowell MS. A case report of neuroleptic malignant syndrome without fever in a patient given aripiprazole. J Okla State Med Assoc 2006;99(7):435-438. 
33. Sullivan CF. A possible variant of the neuroleptic malignant syndrome. Br J Psychiatry 1987;151:689-690.

34. Levenson JL. Neuroleptic malignant syndrome. Am J Psychiatry 1985;142(10):1137-1145.

35. Gibbons GM, Wein DA, Paula R. Profound hypothermia secondary to normal ziprasidone use. Am J Emerg Med 2008;26(6):737 e1-2.

36. Bhanushali MJ, Tuite PJ. The evaluation and management of patients with neuroleptic malignant syndrome. Neurol Clin 2004;22(2):389-411.

37. Strawn J, Keck P, Caroff S. Neuroleptic Malignant Syndrome. Am J Psychiatry 2007;164(6):870-876.

38. Adnet P, Lestavel P, Krivosic-Horber R. Neuroleptic malignant syndrome. Br J Anaesth 2000;85(1):129-135.

39. Sakkas P, Davis JM, Janicak PG, et al. Drug treatment of the neuroleptic malignant syndrome. Psychopharmacol Bull 1991;27(3):381-384.

40. Pope HG, Jr, Aizley HG, Keck PE, Jr, et al. Neuroleptic malignant syndrome: long-term follow-up of 20 cases. J Clin Psychiatry 1991;52(5):208-212.

41. Davis JM, Caroff SN, Mann SC. Treatment of Neuroleptic Malignant Syndrome. Psychiatric Annals 2000;30(5):325-332.

42. Tsutsumi Y, Yamamoto K, Matsuura S, et al. The treatment of neuroleptic malignant syndrome using dantrolene sodium. Psychiatry Clin Neurosci 1998;52(4):433-438.

43. Hamburg P, Weilburg JB, Cassem NH, et al. Relapse of neuroleptic malignant syndrome with early discontinuation of amantadine therapy. Compr Psychiatry 1986;27(4):272-275.
44. Trollor JN, Sachdev PS. Electroconvulsive treatment of neuroleptic malignant syndrome: a review and report of cases. Aust N Z J Psychiatry 1999;33(5):650-659.

45. Boyer EW, Shannon M. The serotonin syndrome. N Engl J Med 2005;352(11):1112-1120.

46. Cheng PL, Hung SW, Lin LW, et al. Amantadine-induced serotonin syndrome in a patient with renal failure. Am J Emerg Med 2008;26(1):112 e5-6.

47. Litman RS, Rosenberg H. Malignant hyperthermia: update on susceptibility testing. JAMA 2005;293(23): 2918-2924.

48. Ali SZ, Taguchi A, Rosenberg H. Malignant hyperthermia. Best Pract Res Clin Anaesthesiol 2003;17(4):519-533.

49. Kao LW, Amin Y, Kirk MA, et al. Intrathecal baclofen withdrawal mimicking sepsis. J Emerg Med 2003;24(4): 423-427.

50. Bianchi M, Castiglioni MG. Refractory generalized edema: an infrequent complication of long-term pergolide treatment for Parkinson disease. Clin Neuropharmacol 2005;28(5):245-246.

51. Blackard WG: Edema-an infrequently recognized complication of bromocriptine and other ergot dopaminergic drugs. Am J Med 1993;94(4):445.

52. Chou KL: Adverse Events from the Treatment of Parkinson's Disease. Neurol Clin 2008;26(3 Suppl):S65-83.

53. American Psychiatric Association. Diagnostic and Statistical Manual of Mental Disorders. 4th ed. Text Revision. Washington DC: American Psychiatric Association; 2000. 